\title{
Three Thirteens
}

\author{
By L. J. Lander
}

Abstract. From a computed solution to the Tarry-Escott problem two sets of thirteen integers are obtained having equal sums of odd powers through the thirteenth.

A recent computer search yielded a solution to the Tarry-Escott problem $\sum_{i=1}^{n} a_{i}^{j}$ $=\sum_{i=1}^{n} b_{i}^{i}, j=1,2, \cdots, k$, with $k=14, n=26$. The terms $a_{1}, a_{2}, \cdots, a_{26}$ are $1,8,9,22,23,34,36,48,50,62,75,83,87,89,95,97,109,130,132,134,136,156$, $157,158,171,173$, and $b_{i}=175-a_{i}, i=1,2, \cdots, 26$. Previously the solution with fewest terms for $k=14$ had $n=30$ [1]. From the new solution it is possible to derive $\sum_{i=1}^{13}\left\{b_{i}-a_{i}\right\}^{x}=\sum_{i=14}^{26}\left\{a_{i}-b_{i}\right\}^{x}$ or $1^{x}+9^{x}+25^{x}+51^{x}+75^{x}+79^{x}+$ $103^{x}+107^{x}+129^{x}+131^{x}+157^{x}+159^{x}+173^{x}=3^{x}+15^{x}+19^{x}+43^{x}+$ $85^{x}+89^{x}+93^{x}+97^{x}+137^{x}+139^{x}+141^{x}+167^{x}+171^{x}$ for $x=1,3,5,7$, $9,11,13$ in which there are 13 terms on each side of the equation.

\section{Control Data Corporation}

4550 West 77th Street

Minneapolis, Minnesota 55435

1. A. Gloden, Mehrgradige Gleichungen, 2nd ed., Noordhoff, Groningen, 1944, p. 58. MR 8, 441.

Received August 31, 1972.

AMS (MOS) subject classifications (1970). Primary 10B15.

Key words and phrases. Thirteenth-order equations, Tarry-Escott problem.

Copyright @ 1973, American Mathematical Society 